\title{
Reliable Centralized Control of PMSM
}

\author{
M. Zazi, Y. Hajji, N. Khaldi, N. Elalami \\ Research Team in Robotics and Control of Linear Systems and Nonlinear \\ (Laboratory of Mechanics, and Industrial Processes) \\ Mohammed V Souissi University \\ Higher School of Technical Education of Rabat, \\ B.P.6207 Avenues des Forces Armées Royales, Rabat 10100, Morocco \\ Tel: +21253771 14 07/08 Fax: +212537711406
}

m.zazi@um5s.net.ma ;youssef.hajji@um5s.net.ma ; naoufel.khaldi@um5s.net.ma ; elalami@emi.ac.ma

Received: April 22, 2021. Revised: Novemeber 15, 2021. Accepted: December 23, 2021. Published: January 10, 2022.

\begin{abstract}
In this paper, we introduce the development methodology of a reliable centralized control applied to a synchronous permanent magnet machine. The proposed system is nonlinear, we linearize around a point of application. The resulting model will then be used to reproduce the dynamic behavior of the machine for a reliable control. The controller is based on the standard $h$ infinite to increase performance, reduce measurement noise, and to tolerate the outage of certain sensors. To illustrate the results, we made a comparison between a standard state feedback control and reliable $h$ infinite robust control. The simulation results shows, that the system in case of technical placements poles loses classic performance in the presence of an outage, that the reliable centralized robust control remain satisfactory performance even in the presence of outage.
\end{abstract}

Keywords-Reliable control, $\boldsymbol{H}$ infinite, Equation of Riccati, linear system, state feedback control, centralized control, reliable centralized control.

\section{INTRODUCTION}

In the presence of disturbances, the technique of classical state feedback control of linear systems appears insufficient and gives mediocre performance. But in the case of sensor outage it's clearly that the system loses its performances, researches propose adaptive algorithms.

The main advantage of Reliable Centralized Control techniques is to generate control laws which allow firstly to shape the response of the servo system to give it the desired behavior and secondly maintain this behavior to the vagaries and fluctuations that affect even in the presence of sensor outage.

In this paper we investigate a control strategy which is to develop centralized controller based on the synthesis of $\mathrm{H}$ infinite and having the structure of a state observer estimates increased disturbances and commands generated by other controllers so to ensure a stable transfer between magnitudes and bounded external disturbances regulated and the acting on the whole system. The method is therefore primarily to solve a Riccati equation. We conclude by validating the results using a simulation that compares the standard state feedback controller and the reliable centralized control.

\section{DESIGN OF RELIABle CENTRALIZED Control [1] $[10][13]$}

In this section we introduce the problem of designing a centralized controller that is reliable despite possible sensor

outages. The outages will be restricted of occur within a preselected subset of available measurements .The controllers developed will guarantee closed-loop stability and prescribed

$\mathrm{H} \infty$-norm bound, regardless of admissible sensor failures.

Consider first the design of a controller that can tolerate the outage of certain sensors. Let $\Omega \subseteq\{1,2, \ldots, \operatorname{dim}(y)\}$ correspond to a selected subset of sensors susceptible to outages. Introduce the decomposition

$C=C_{\Omega}+C_{\bar{\Omega}}$

Where $C_{\Omega}$ denotes the measurement matrix associated with $\Omega$, and $C_{\bar{\Omega}}$ denotes the measurement matrix associated with the complementary subset of measurements .to explain, $C_{\bar{\Omega}}$ is formed from $\mathrm{C}$ by putting zeros out rows corresponding to susceptible sensors .Let $w \subseteq \Omega$ correspond to a particular subset of the susceptible sensors that actually experience an outage, and let $T_{\bar{w}}(s)$ denote the transfers-function matrix of the resulting closed-loop system. We can adopt the notation $C=C_{\mathrm{w}}+C_{\overline{\mathrm{w}}}$

Where $C_{\mathrm{w}}$ and $C_{\overline{\mathrm{w}}}$ have meaning analogous to those of $C_{\Omega}$ and $C_{\bar{\Omega}}$ in (1). Since $w \subseteq \Omega \quad, C_{w}^{T} C_{w} \leq C_{\Omega}^{T} C_{\Omega}$. Also, decompose the observer gain as

$L=L_{\mathrm{w}}+L_{\overline{\mathrm{w}}}$

So that $L C=L_{\mathrm{w}} C_{\mathrm{w}}+L_{\overline{\mathrm{w}}} C_{\overline{\mathrm{w}}}$

(That is, $L_{\overline{\mathrm{w}}}$ has zero columns corresponding to sensors which have actually failed) Then the following result hold.

THEOREM4.1: with all assumptions and the design otherwise as in corollary 2.4[1], assume $X \geq 0$ and $Y>0$ satisfy the ARE's

$$
\begin{aligned}
& A^{T} X+X A-X S X+\frac{1}{\alpha^{2}} X G G^{T} X+H^{T} H+\alpha^{2} C_{\Omega}^{T} C_{\Omega}=0 \\
& A Y+Y A^{T}+\frac{1}{\alpha^{2}} Y H H^{T} Y-Y C_{\bar{\Omega}}^{T} C_{\bar{\Omega}} Y+G G^{T}=0
\end{aligned}
$$

Respectively. Then, for sensor outages corresponding to any $w \subseteq \Omega$, the closed-loop system is stable, and $\left\|T_{\bar{w}}\right\|_{\infty} \leq \alpha$.

Remarks:

1) with all sensors operational, which corresponds to $w=\emptyset, T_{\bar{w}}(s)=T(s)$ is the, nominal closed-loop transferfunction matrix from $w_{e}$ to $\mathrm{z}$, where

$$
w_{e}=\left(\begin{array}{c}
w_{0} \\
w
\end{array}\right), z=\left(\begin{array}{c}
H x \\
u
\end{array}\right)
$$


Theorem 4.1 covers this case automatically, since $w=\emptyset \subseteq$ $\Omega$. If sensors corresponding to a nonempty subset $w \subseteq \Omega$ fail, then $T_{\bar{w}}(s)$ is the resulting transfer-function matrix from $w_{e \bar{w}}$ to $\mathrm{z}$, where

$$
w_{e \bar{w}}=\left(\begin{array}{l}
w_{0} \\
w_{\bar{w}}
\end{array}\right)
$$

With $w_{\bar{w}}$ containing only those components of measurement noise associated with operational sensors, thus, for the present reliability formulation, a sensor failure effectively eliminates the associated sensor noise.

2) The design equation (5) and (6) arise from replacing $H$ in the description of the plant by the augmented matrix.

$H_{+}=\left(\begin{array}{c}H \\ \alpha C_{\Omega}\end{array}\right)$

And changing the basic design equations accordingly. This corresponds to choosing the matrix $P e$ in (2) as

$$
P e=\alpha^{2}\left(\begin{array}{cc}
C_{\Omega}^{T} C_{\Omega} & 0 \\
0 & 0
\end{array}\right) \geq 0
$$

Proof: In view of remark 2) above, if (5) and (6) have appropriate solution, then corollary $2.4[1]$ guarantees that $X e \geq 0$ satisfies.

$F_{e}^{T} X_{e}+X_{e} F_{e}+\frac{1}{\alpha^{2}} X_{e} G_{e} G_{e}^{T} X_{e}+H_{e+}^{T} H_{e+}=0$

And that $\left(F_{e}, H_{e+}\right)$ is a detectable pair, where the augmented closed-loop system is described by the matrices

$$
\begin{aligned}
& F_{e}=\left(\begin{array}{cc}
A & B K \\
L C & A_{\alpha}-L C
\end{array}\right), G_{e}=\left(\begin{array}{ll}
G & 0 \\
0 & L
\end{array}\right) \\
& H_{e+}=\left(\begin{array}{cc}
H_{+} & 0 \\
0 & K
\end{array}\right)
\end{aligned}
$$

The actual closed-loop system with no sensor outages is described by the matrices

$$
\begin{aligned}
& F_{e}=\left(\begin{array}{cc}
A & B K \\
L C & A_{\alpha}-L C
\end{array}\right), G_{e}=\left(\begin{array}{cc}
G & 0 \\
0 & L
\end{array}\right) \\
& H_{e}=\left(\begin{array}{cc}
H & 0 \\
0 & K
\end{array}\right)
\end{aligned}
$$

For sensor outages corresponding to $w \subseteq \Omega$, the controller effectively becomes

$\dot{\xi}=\left(A+B K+G K_{d}-L c\right) \xi+L_{\bar{w}} y$

$u=K \xi$

The controller dynamic structure is not affected by sensor outages; only the controller input structure is effectively changed. Given (11) and (12), the closed-loop system matrices become.

$$
\begin{aligned}
F_{e \bar{w}} & =\left(\begin{array}{cc}
A & B K \\
L_{\bar{w}} C_{\bar{w}} & A_{\alpha}-L C
\end{array}\right), G_{e \bar{w}}=\left(\begin{array}{cc}
G & 0 \\
0 & L_{\bar{w}}
\end{array}\right) \\
H_{e} & =\left(\begin{array}{cc}
H & 0 \\
0 & K
\end{array}\right)
\end{aligned}
$$

It follows that

$F_{e}=F_{e \bar{w}}+\left(\begin{array}{c}0 \\ L_{w}\end{array}\right)\left(\begin{array}{ll}C_{w} & 0\end{array}\right) \equiv F_{e \bar{w}}+L_{e w} C_{e w}$

$G_{e} G_{e}^{T}=\left(\begin{array}{cc}G G^{T} & 0 \\ 0 & L_{\bar{w}} L_{\bar{w}}^{T}\end{array}\right)+\left(\begin{array}{c}0 \\ L_{w}\end{array}\right)\left(\begin{array}{ll}0 & L_{w}^{T}\end{array}\right) \equiv G_{e \bar{w}} G_{e \bar{w}}^{T}+L_{e w}+$

$L_{e w}^{T}$

$H_{e+}^{T} H_{e+}=H_{e}^{T} H_{e}+\alpha^{2}\left(\begin{array}{cc}C_{\Omega}^{T} C_{\Omega} & 0 \\ 0 & 0\end{array}\right)$

Use (14) in (8) and the fact, $C_{w}^{T} C_{w} \leq C_{\Omega}^{T} C_{\Omega}$ to obtain

$$
\begin{aligned}
F_{e \bar{w}}^{T} X_{e}+X_{e} F_{e \bar{w}}+ & \frac{1}{\alpha^{2}} X_{e} G_{e \bar{w}} G_{e \bar{w}}^{T} X_{e}+H_{e+}^{T} H_{e+} \\
& =-C_{e w}^{T} L_{e w}^{T} X_{e}-X_{e} L_{e w} C_{e w} \\
& +\frac{1}{\alpha^{2}} X_{e} L_{e w} L_{e w}^{T} X_{e}-\alpha^{2}\left(\begin{array}{c}
C_{\Omega}^{T} \\
0
\end{array}\right)\left(\begin{array}{ll}
C_{\Omega} & 0
\end{array}\right) \\
& \leq-C_{e w}^{T} L_{e w}^{T} X_{e}-X_{e} L_{e w} C_{e w} \\
& -\frac{1}{\alpha^{2}} X_{e} L_{e w} L_{e w}^{T} X_{e}-\alpha^{2} C_{e w}^{T} C_{e w} \\
& =-\left(\frac{1}{\alpha} X_{e} L_{e w}+\alpha C_{e w}^{T}\right)\left(\frac{1}{\alpha} L_{e w}^{T} X_{e}+\alpha C_{e w}\right) \\
& \leq 0
\end{aligned}
$$

Hence, provided $\left(F_{e \bar{w}}, H_{e}\right)$ is a detectable pair, see lemma 2.1 [1] guarantees that $F_{e \bar{w}}$ is Hurwitz, and $\operatorname{that} T_{\bar{w}}(s)=$ $H_{e}\left(s I-F_{e \bar{w}}\right)^{-1} G_{e \bar{w}}$, the transfer-function matrix from $w_{e \bar{w}}$ to $\mathrm{z}$, satisfies $\left\|T_{\bar{w}}\right\|_{\infty} \leq \alpha$. The detectability proof is routine: if $v^{T}=\left(\begin{array}{cc}v_{1}^{T} & v_{2}^{T}\end{array}\right) \neq 0$ satisfies $F_{e \bar{w}} v=\lambda v$ and $H_{e} v=0$, then $A v_{1}=\lambda v_{1}$, And $H v_{1}=0$, with $(\mathrm{A}, \mathrm{H})$ assumed a detectable pair, Therefore, either $\operatorname{Re}(\lambda)<0$ or $v_{1}=0$. If $v_{1}=0$, then $F_{e} v=F_{e \bar{w}} v=\lambda v$ and $H_{e} v=0$ gives $H_{e+} v=0$. Since $\left(F_{e}, H_{e+}\right)$ is a detectable pair, $\operatorname{Re}(\lambda)<0$ Q.E.D

\section{MODELING OF THE PMSM}

The machine can be represented by the shape of state space and can be written [2], [3]:

With:

$$
\left\{\begin{array}{l}
X=A(\dot{x})+B u \\
Y=C(x)
\end{array}\right.
$$

$\dot{x}_{1}=\frac{d}{d t} i_{d}=-\frac{R}{L_{d}} i_{d}+\frac{L_{q}}{L_{d}} P \Omega i_{q}+\frac{u_{d}}{L_{d}}$

$\dot{x}_{2}=\frac{d}{d t} i_{q}=-\frac{R}{L_{q}} i_{q}-\frac{L_{d}}{L_{q}} P \Omega i_{d}-\frac{\phi f}{L_{q}} P \Omega+\frac{u_{q}}{L_{q}}$

$\dot{x}_{3}=\frac{d}{d t} \Omega=\frac{P}{J}\left[\phi f i_{q}+\left(L_{d}-L_{q}\right) i_{d} i_{q}\right]-\frac{f}{J} \Omega$

The system is rewritten as desired linearization [11], [12][13]:

$$
\begin{gathered}
x=\left[\begin{array}{l}
x_{1} \\
x_{2} \\
x_{3}
\end{array}\right]=\left[\begin{array}{l}
i_{d} \\
i_{q} \\
\Omega
\end{array}\right] ; u=\left[\begin{array}{l}
u_{d} \\
u_{q}
\end{array}\right] \\
F(x)=\left[\begin{array}{l}
f_{1}(x) \\
f_{2}(x) \\
f_{3}(x)
\end{array}\right]=\left[\begin{array}{c}
a_{1} x_{1}+a_{2} x_{2} x_{3} \\
b_{1} x_{2}+b_{2} x_{1} x_{3}+b_{3} x_{3} \\
c_{1} x_{3}+c_{2} x_{1} x_{2}+c_{3} x_{2}
\end{array}\right]
\end{gathered}
$$

With:

$$
\begin{aligned}
& a_{1}=-\frac{R}{L_{d}} ; a_{2}=\frac{L_{q}}{L_{d}} P \\
& b_{1}=-\frac{R}{L_{q}} ; b_{2}=-\frac{L_{d}}{L_{q}} P ; b_{3}=-\frac{\phi f}{L_{q}} P \\
& c_{1}=-\frac{f}{J} ; c_{2}=\frac{P}{J}\left(L_{d}-L_{q}\right) ; c_{3}=\frac{P}{J} \phi f
\end{aligned}
$$

The following parameters [2] of the PMSM are:

$\mathrm{Ld}=11 \mathrm{mH} ; \mathrm{Lq}=11 \mathrm{mH} ; \phi \mathrm{f}=0.18 \mathrm{wb} ; \mathrm{J}=6 \mathrm{e}^{-4} \mathrm{Kg} . \mathrm{m}^{2}$

$\mathrm{F}=1 \mathrm{e}^{-4} \mathrm{~N} . \mathrm{m} . \mathrm{s} / \mathrm{rad} ; \mathrm{R}=1.2 \boldsymbol{\Omega} ; \mathrm{P}=3$

This model is linearized about an operating point: 


$$
x_{0}=\left[\begin{array}{l}
i_{d 0} \\
i_{q 0} \\
\Omega_{0}
\end{array}\right] ; u_{0}=\left[\begin{array}{l}
u_{d 0} \\
u_{q 0}
\end{array}\right]
$$

We can correspond those matrixes to our system:

$$
\begin{aligned}
& \dot{x}=A x+B u+G w_{0} \\
& y=C x+w \\
& z=\left[\begin{array}{c}
H x \\
u
\end{array}\right]
\end{aligned}
$$

Which gives us:

A

$$
\begin{aligned}
& =\left[\begin{array}{ccc}
-\frac{R}{L_{d}} & \frac{L_{q}}{L_{d}} P \Omega_{0} & \frac{L_{q}}{L_{d}} P i_{q 0} \\
-\frac{L_{d}}{L_{q}} P \Omega_{0} & -R / L_{q} & -\frac{L_{d}}{L_{q}} P i_{d}-\frac{\phi f P}{L_{q}} \\
\frac{P}{J}\left(\left(L_{d}-L_{q}\right) i_{q}\right. & \frac{P}{J}\left(\phi f+\left(L_{d}-L_{q}\right) i_{d}\right) & -\frac{f}{J}
\end{array}\right] \\
& B=\left[\begin{array}{cc}
1 / L_{d} & 0 \\
0 & 1 / L_{q} \\
0 & 0
\end{array}\right] ; C=\left[\begin{array}{ccc}
0 & 0 & 1 \\
1 & 0 & 0
\end{array}\right] \\
& H=\left[\begin{array}{ccc}
0 & 0 & 1
\end{array}\right] ; G=\left[\begin{array}{c}
0 \\
0 \\
-\frac{1}{J}
\end{array}\right] C_{r}
\end{aligned}
$$

Finally, the operating point and the vector control selected are:

$$
\left[\begin{array}{l}
i_{d 0} \\
i_{q 0} \\
\Omega_{0}
\end{array}\right]=\left[\begin{array}{c}
0 \\
0 \\
50
\end{array}\right] ;\left[\begin{array}{l}
u_{d 0} \\
u_{q 0}
\end{array}\right]=\left[\begin{array}{c}
0 \\
27
\end{array}\right]
$$

\section{APPLICATION OF CONTROLS}

\section{A. Application of state feedback control:}

The choice of the gain matrix $\mathrm{K}$ based on the location of the poles that wants to impose the system, we take the following clusters [2], [9]:

$$
[-10,-0.125,-4000]
$$

To obtain the gain matrix $\mathrm{K}$ :

$$
\mathrm{K}=\left[\begin{array}{ccc}
-1.1987 & 1.6515 & 0.0003 \\
-1.6489 & 42.9125 & -0.0593
\end{array}\right]
$$

\section{B. Application of $H \infty$ Algorithm:}

By applying the algorithm previously mentioned we come to find the values of $\mathrm{X}$ which we calculate the value of gains $\mathrm{K}$, $\mathrm{Kd}$, and $\mathrm{Li}$ which are the synthesis parameters of the law.

We have:

$$
\mathrm{K}=-\mathrm{B}^{\mathrm{T}} \mathrm{X} ; \mathrm{K}_{\mathrm{d}}=\frac{1}{\alpha^{2}} \mathrm{G}^{\mathrm{T}} \mathrm{X}
$$

$\mathrm{L}$ is obtained by resolving the Riccati equation which returns $\mathrm{Y}$.

$$
L=\left(I-\alpha^{-2} Y X\right)^{-1} Y C^{T}
$$

Without an outage we have:

$$
\begin{gathered}
K=\left[\begin{array}{crr}
-0.5982 & 0.6862 & 0.1104 \\
0.6862 & -2.0246 & -0.5906
\end{array}\right] \\
K_{d}=\left[\begin{array}{lrr}
-0.0304 & 0.1624 & 0.0905
\end{array}\right] \mathrm{e}^{-4} \\
L=\left[\begin{array}{rr}
-0.0002 & 0.0001 \\
-0.0006 & 0.0001 \\
0.0045 & -0.0002
\end{array}\right]
\end{gathered}
$$

With an outage on speed sensor we have:

$$
\begin{gathered}
K=\left[\begin{array}{crr}
-0.5982 & 0.6862 & 0.1104 \\
0.6862 & -2.0246 & -0.5906
\end{array}\right] \\
K_{d}=\left[\begin{array}{lrr}
-0.0304 & 0.1624 & 0.0905
\end{array}\right] \mathrm{e}^{-4} \\
L=\left[\begin{array}{rr}
-0.0002 & 0.0001 \\
-0.0006 & 0.0001 \\
0.0045 & -0.0002
\end{array}\right]
\end{gathered}
$$

With reliable we have:

$$
\begin{gathered}
K=\left[\begin{array}{ccc}
-0.8382 & 1.5571 & 0.4770 \\
1.5571 & -18.2852 & -19.4829
\end{array}\right] \\
K_{d}=\left[\begin{array}{llr}
0.000 & 0.0005 & 0.0011
\end{array}\right] \\
L=\left[\begin{array}{rr}
-0.0002 & 0.0001 \\
-0.0006 & 0.0001 \\
0.0045 & -0.0002
\end{array}\right]
\end{gathered}
$$

Between the three cases, the value of the L matrix doesn't change, while for $\mathrm{Kd}$ it varies weakly, the only big changeover is to the level of the $\mathrm{K}$ matrix

\section{SimUlation}

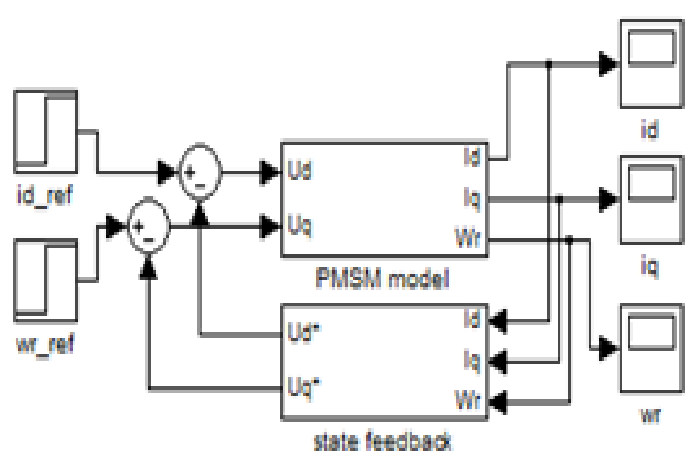

FigV.1: PMSM with state feedback controller 


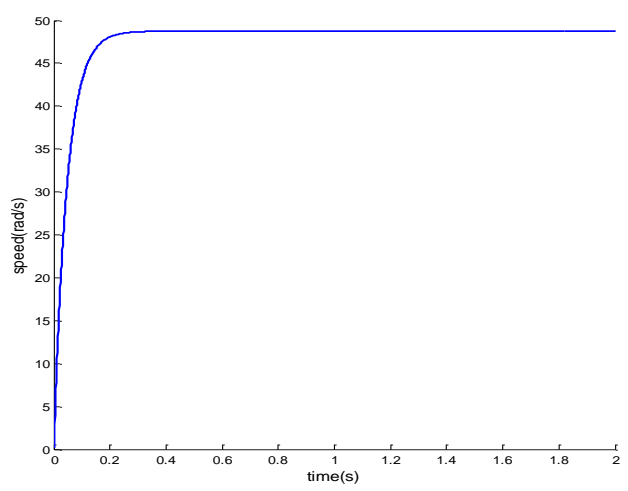

FigV.2:speed with state feedback without noise

With absence of noise the speed of the PMSM can reach the reference value with a static error which can be removed with a pre-compensator gain.

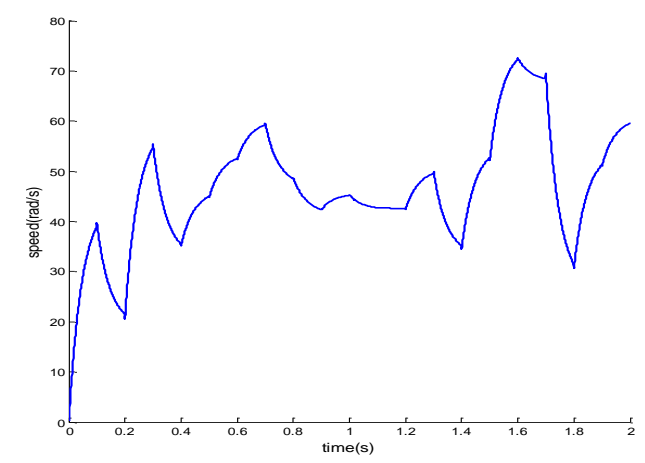

FigV.3:speed with state feedback with 0.01 noise power without failure

In the presence of noise the PMSM with state feedback controller lose its performance, and become unstable.

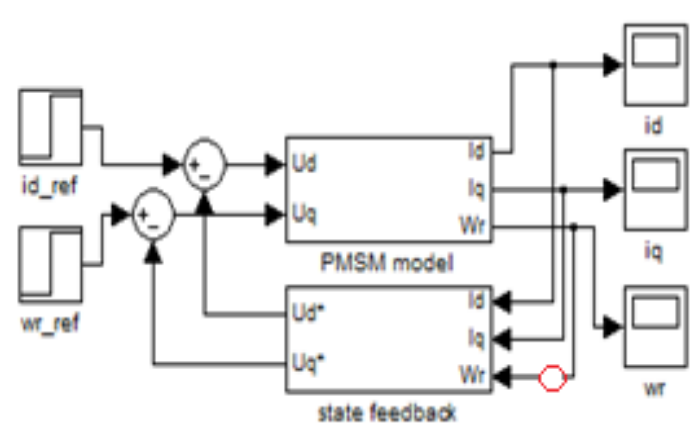

FigV.4 PMSM with state feedback controller with failure on speed sensor

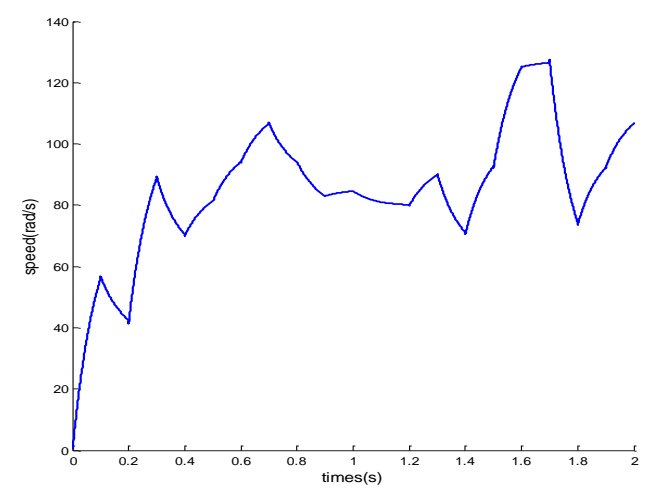

FigV.5:speed with state feedback with 0.01 noise power with failure on speed sensor

With failure on the speed sensor the PMSM loses definitely its performances.

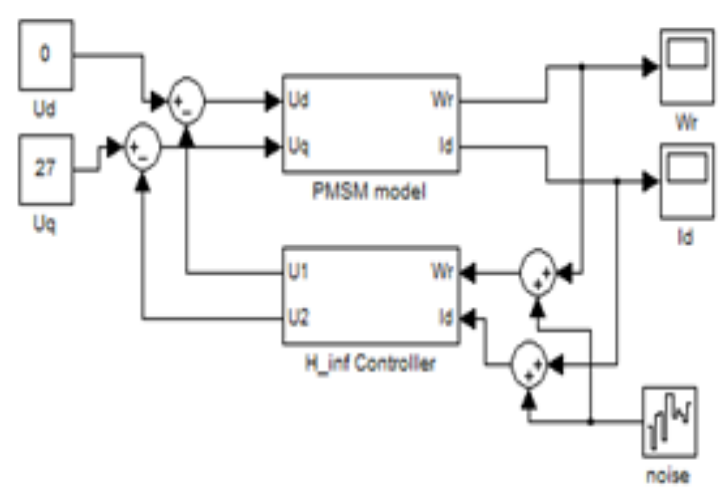

FigV.6:PMSM with $\mathrm{H} \infty$ controller

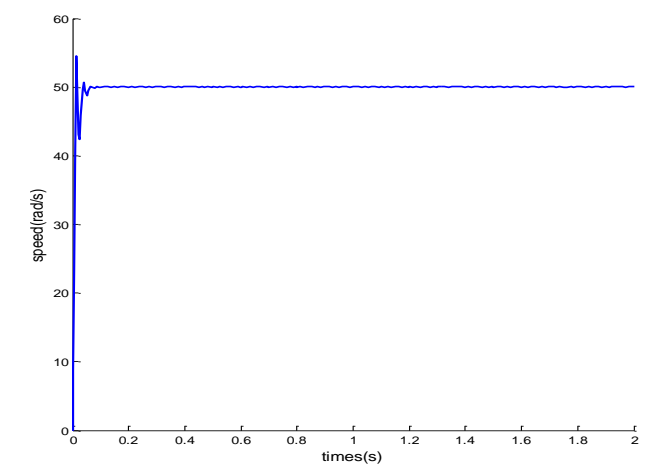

FigV.7:speed with standard $\mathrm{H} \infty$ controller without noise 


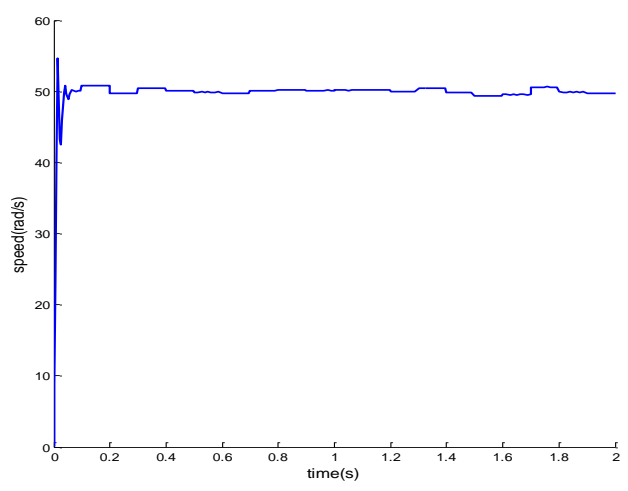

FigV.8:speed with $\mathrm{H} \infty$ controller with 0.01 noise power without failure

The $\mathrm{H} \infty$ controller minimizes the effect of the noise on the speed of the PMSM.

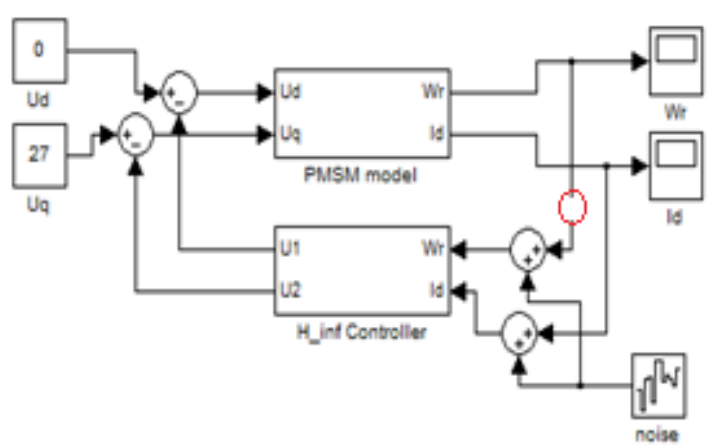

FigV.9: PMSM with $\mathrm{H} \infty$ controller with failure on speed sensor

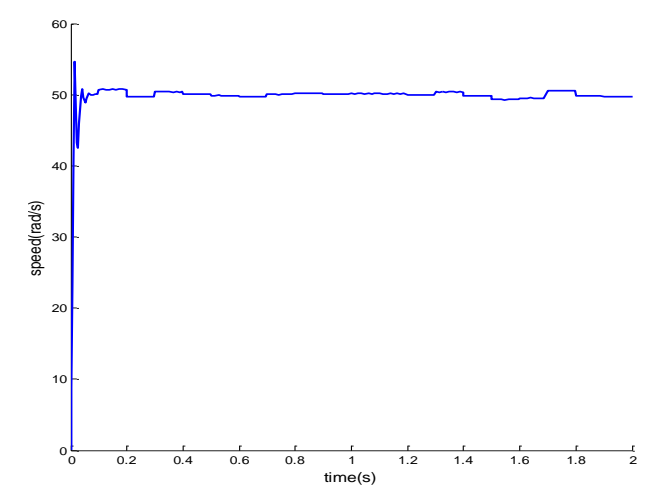

FigV.10: speed with $\mathrm{H} \infty$ controller whit 0.01 noise power with failure on speed sensor

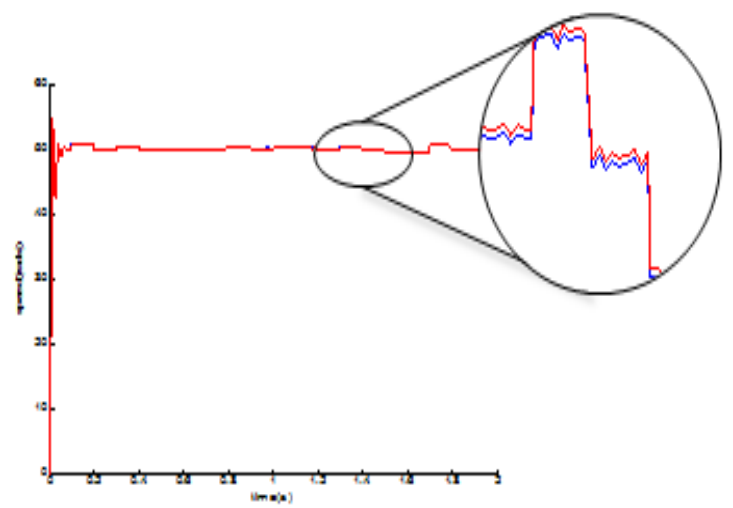

figV.11:comparaison of speed with $\mathrm{H} \infty$ controller with 0.01 noise power(failure \& safe)

In the case of an outage on speed sensor the performances deteriorate but not clearly (in red safe case, in blue with failure)

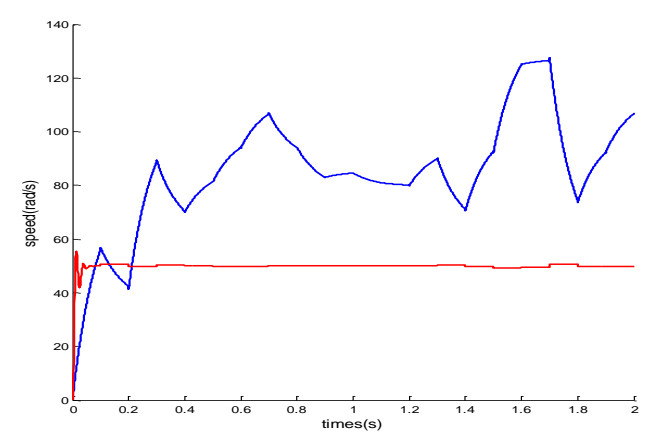

figV.11:comparaison of speed with $\mathrm{H} \infty$ controller and standard state feedback with 0.01 noise power with failure on speed sensor

\section{CONCLUSION}

The works presented in this paper have been devoted to the study of the reliable Centralized Control applying to a PMSM.

A comparison of the changes in engine speed when applying a classical control state feedback and $\mathrm{H} \infty$ centralized control.

The controller Ho centralized is known for its hardiness, so it maintains the steady system even in the presence of an outage on sensor.

\section{REFERENCES}

[1] Veillette, R.J., Design of reliable control system, IEEE transactions on automatic control. Vol 37.NO.3.MARCH 1992.

[2] KADOURRI Azzedine, Etude d'une commande non linéaire adaptative d'une machine synchrone à aimants permanents, Thèse de doctorat, FACULTÉ DES SCIENCES ET DE GÉNIE UNIVERSITÉ LAVAL, QUÉBEC, Année 2000.

[3] BENBRAHIM Amel, Commande prédictive généralisée d'une machine synchrone à aimants permanents, mémoire de magister, Université de Batna, Algérie, Année 2009. 
[4] T.C. Minh, and L.H. Hoang "Model reference adaptive fuzzy controller and fuzzy estimator for high performance induction motor drives" Proceedings of the annual meeting of the IEEE Industry Applications society, California, 1996.

[5] R. Marino, S. Peresada, and P. Valigi "Adaptive input output linearizing control of induction motor" IEEE. Trans. Automat. Contr. Vol.38, No2, pp 208-221, 1993

[6] A.Titaouine,F.Benchabane,K.Yahia.A.Moussi ,,commande d'une machine synchrone a aimants permanents et estimation de ces parametres en utilisant le filtre de KALMAN etendu ${ }^{\text {ee }}$ Courrier du savoir-N7, décembre 2006,pp37-43.

[7] Dongemei X. ,Q.Daokui et X.Fang "Design of Ho feedback controller and IP position controller of PMSM servo system",IEEE Transactions on Industrial Electronices, vol 48,no.6,december 2005,pp.1098-1108

[8] Hsien T.-L.,Y.Y. Sun et M. C. Tsai "Hळ control for sensorless permanent-magnet synchronous drive", IEEE Transactions on Industrial Electronics, vol 144,no.3,may 1997,pp.173-181 M. Young, The Technical Writers Handbook. Mill Valley, CA: University Science, 1989.

[9] Anurag Kumar Swami "A robust optimal state feedback control design" 6th WSEAS international conference on system science and simulation in engineering, Venice, Italy, November 21-23, 2007.

[10] Kamuran Turkoglu, Elbrous M. Jafarov " $H$ inf. loop shaping robust control vs. Classical PI(D) Control: a case study on the longitudinal dynamics of hezarfen UAV "proceedings of the 2nd WSEAS international conference on dynamical systems and control, Bucharest, Romania, October 16-17,2006.

[11] A.NAIT SEGHIR and M.S.BOUCHERIT" Artificial Neural Network to Improve Speed Control of Permanent Magnet Synchronous Motor" Proceedings of the 6th WSEAS/IASME int. conf. on electric power systems, high voltages, electric machines, Tenerife, Spain December 16-18,2006

[12] L. Farah, N. Farah, M. Bedda "Control of induction Motor Drive by Artificial Neural Network" proc. of the 5th WSEAS/IASME int. conf. on electric power systems, high voltages, electric machines, Tenerife, Spain, December 16-18,2005.

[13] Y.HAJJI,N.KHALDI,M.ZAZI,N.ELALAMI"Modelind and Robust Hळ control of linear systems" proc.of the $13^{\text {th }}$ WSEAS International Conference on SIGNAL PROCESSING,ROBOTICS and AUTOMATION(ISPRA“13)Cambridge, UK, February 20-22,2013

\section{Creative Commons Attribution License 4.0 (Attribution 4.0 International, CC BY 4.0)}

This article is published under the terms of the Creative Commons Attribution License 4.0 https://creativecommons.org/licenses/by/4.0/deed.en US 